\title{
HIGHER HOMOTOPY COMMUTATIVITY AND EXTENSION OF MAPS
}

\author{
F. D. WILLIAMS
}

Abstract. Let $X$ denote the cartesian product of based spaces, $\mathrm{X}=X_{1} \times \cdots \times X_{n}$, and $A=X_{1} \vee \cdots \vee X_{n}$, the subspace consisting of their one-point union. Further, let $g: A \rightarrow Y$ be a map, for $Y$ any based space. This article develops a criterion for the extendibility of $g$ to a map $G: X \rightarrow Y$. The criterion is in terms of higher products which live in the Pontryagin ring of $\Omega Y$, the loop space of $Y$.

A basic problem in topology is the extension problem: Given spaces $A, X$, and $Y$, where $A$ is a subspace of $X$, and a map $g: A \rightarrow Y$, can $g$ be extended to a map $G: X \rightarrow Y$ ? In the category of based spaces, an important special case is that in which $X$ is the cartesian product $X_{1} \times \cdots \times X_{n}$ of spaces $X_{1}, \cdots, X_{n}$ and $A=X_{1} \vee \cdots \vee X_{n}$, the subspace consisting of their one-point union. In [6], Samelson treated this case for $n=2$ and $X_{1}$ and $X_{2}$ being spheres, by relating the extension problem to the Pontryagin product in the homology of $\Omega Y$, the loop space of $Y$. In this note, Samelson's method is extended to the general situation of arbitrary $X_{1}, \cdots, X_{n}$ through the use of the higher homotopy commutativity introduced in [7]. The outline is as follows. The first section transforms the extension problem to a problem in higher topological homotopy commutativity, and the second section completes the transfer to a problem in higher algebraic homotopy commutativity. The final section illustrates the method through an application to complex Grassmann manifolds. The author wishes to acknowledge helpful suggestions from G. J. Porter.

1. We begin by recalling the basic definitions of higher homotopy commutativity.

Let $K_{n}$ denote the $(n-1)$-dimensional convex linear cell obtained by taking the convex hull of the orbit of the point $(1, \cdots, n)$ under permutation of the coordinates in $R^{n}$. If $0<r<n$, define $d_{r}: K_{r} \times K_{n-r}$ $\rightarrow K_{n}$ by

$$
d_{r}\left(x_{1}, \cdots, x_{r}, y_{1}, \cdots, y_{n-r}\right)=\left(x_{1}, \cdots, x_{r}, y_{1}+r, \cdots, y_{n-r}+r\right) .
$$

The (n-2)-dimensional faces of $K_{n}$ are in 1:1 correspondence with the

Received by the editors November 21, 1969.

AMS 1969 subject classifications. Primary 5540, 5542; Secondary 5560, 5745.

Key words and phrases. Homotopy commutativity, loop space, suspension, $H$ space, homology ring, higher products, Grassmann manifold. 
set of $(p, q)$-shuffles, $p+q=n$. If $\left(A_{p}, B_{q}\right)$ is such a shuffle, then $V\left(A_{p}, B_{q}\right): K_{p} \times K_{q} \rightarrow K_{n}$ is the homeomorphism given by $V\left(A_{p}, B_{q}\right)$ $=\alpha^{-1} \circ d_{p}$ where $\alpha$ is the homeomorphism of $R^{n}$ to itself determined by the action of $\left(A_{p}, B_{q}\right)$ on the coordinates. For pictures of $K_{n}$, as well as further information about the $K_{n}$ 's, see [3].

Definition 1. Let $G$ be an associative $H$-space and $Z_{1}, \cdots, Z_{n}$ be pointed spaces. For each $i$, let $f_{i}: Z_{i} \rightarrow G$ be a map. Then a $C_{n}$-form for the $f_{i}$ 's consists of a system of maps $f\left(i_{1}, \cdots, i_{p}\right): K_{p} \times Z_{i_{1}} \times \cdots$ $\times Z_{i_{p}} \rightarrow G$, indexed by subsequences $\left(i_{1}, \cdots, i_{p}\right)$ of $(1, \cdots, n)$ such that

$$
f(i)=f_{i},
$$

and

$$
\begin{aligned}
f\left(i_{1}, \cdots, i_{p}\right)\left[V\left(A_{r}, B_{s}\right)\right. & \left.(\rho, \sigma), x_{1}, \cdots, x_{p}\right] \\
= & f\left(i_{A(1)}, \cdots, i_{A(r)}\right)\left[\rho, x_{A(1)}, \cdots, x_{A(r)}\right] \\
& \cdot f\left(i_{B(1)}, \cdots, i_{B(s)}\right)\left[\sigma, x_{B(1)}, \cdots, x_{B(s)}\right],
\end{aligned}
$$

where $\left(A_{r}, B_{s}\right)$ is an $(r, s)$-shuffle of $(1, \cdots, p),(\rho, \sigma) \in K_{r} \times K_{s}$, and $x_{k} \in Z_{i_{k}}$.

REMARKs. Observe that a $C_{2}$-form for two maps $f_{1}$ and $f_{2}$ is just a homotopy between the product maps $f_{1} \cdot f_{2}$ and $f_{2} \cdot f_{1}$. For further intuition into $C_{n}$-commutativity, the reader is referred to p. 193 of [7]. In that paper is defined the notion of $C_{n}$-space: An associative $H$-space is a $C_{n}$-space provided that there is a $C_{n}$-form for the maps $f_{1}, \cdots, f_{n}$ where each $f_{i}=1: G \rightarrow G$. Clearly, if $G$ is a $C_{n}$-space, then any maps $f_{i}: Z_{i} \rightarrow G, i=1, \cdots, n$, possess a $C_{n}$-form.

Now consider maps $\tilde{f}_{i}: S Z_{i} \rightarrow Y, i=1, \cdots, n$, where $S Z_{i}$ denotes the suspension of the space $Z_{i}$. Then the map $\tilde{f}: S Z_{1} \bigvee \cdots \vee S Z_{n} \rightarrow Y$ is defined by $\bar{f} \mid S Z_{i}=\tilde{f}_{i}$. Suppose that $f$ extends to $F: S Z_{1} \times \cdots \times S Z_{n}$ $\rightarrow Y$. Then for each subsequence $\left(i_{1}, \cdots, i_{p}\right)$ of $(1, \cdots, n)$ a map $F\left(i_{1}, \cdots, i_{p}\right): I^{p} \times Z_{i_{1}} \times \cdots \times Z_{i_{p}} \rightarrow Y$ is defined by the composition

$$
\begin{aligned}
I^{p} \times Z_{i_{1}} \times \cdots \times Z_{i_{p}} & \stackrel{q}{\rightarrow} I \times Z_{i_{1}} \times \cdots \times I \times Z_{i_{p}} \\
& \stackrel{r}{\rightarrow} S Z_{i_{1}} \times \cdots \times S Z_{i_{p}} \stackrel{s}{\rightarrow} Y,
\end{aligned}
$$

where $q$ interchanges the factors, $r$ is the identification map, and $s=F \mid S Z_{i_{1}} \times \cdots \times S Z_{i_{p}}$. Now let $\hat{f}\left(i_{1}, \cdots, i_{p}\right):[0, p] \times K_{p} \times Z_{i_{1}}$ $\times \cdots \times Z_{i_{p}} \rightarrow Y$ be defined as follows.

First, let maps $\tau_{p}:[0, p] \times K_{p} \rightarrow I^{p}$ be defined inductively as follows. Let $\tau_{1}:[0,1] \times K_{1} \rightarrow I$ be the identity map $\tau_{1}\left(t,{ }^{*}\right)=t$. Suppose $\tau_{k}$ is 
defined for $k<p$. Define $\tau_{p}$ from $\operatorname{Bd}\left([0, p] \times K_{p}\right) \rightarrow \operatorname{Bd}\left(I^{p}\right)$ by $\tau_{p}(0, \kappa)$ $=(0, \cdots, 0), \tau_{p}(1, \kappa)=(1, \cdots, 1)$, and

$$
\begin{aligned}
\tau_{p}\left(t, V\left(A_{r}, B_{s}\right)(\rho, \sigma)\right) & =\left[A_{r}, B_{s}\right]\left(\tau_{r}(t, \rho), 0, \cdots, 0\right), & & \text { for } 0 \leqq t \leqq r, \\
& =\left[A_{r}, B_{s}\right]\left(1, \cdots, 1, \tau_{s}(t-r, \sigma)\right), & & \text { for } r \leqq t \leqq p,
\end{aligned}
$$

where $\kappa \in K_{p},(\rho, \sigma) \in K_{r} \times K_{s}, t \in[0, p]$, and $\left[A_{r}, B_{s}\right]$ denotes the shuffle acting on $I^{p}$ by permuting the coordinates. The definition of $\tau_{p}$ is completed by regarding $[0, p] \times K_{p}$ and $I^{p}$ as the cones on their respective boundaries and extending $\tau_{p}$ by linearity. Now let $\hat{f}\left(i_{1}, \cdots, i_{p}\right)$ be defined to be the composition $F\left(i_{1}, \cdots, i_{p}\right) \circ$ $\left(\tau_{p} \times\right.$ id $)$. Finally, define $f\left(i_{1}, \cdots, i_{p}\right): K_{p} \times Z_{i_{1}} \times \cdots \times Z_{i_{p}} \rightarrow \Omega Y$ by the formula

$$
f\left(i_{1}, \cdots, i_{p}\right)\left(\tau, z_{i_{1}}, \cdots, z_{i_{p}}\right)[t]=\hat{f}\left(i_{1}, \cdots, i_{p}\right)\left(t, \tau, z_{i_{1}}, \cdots, z_{i_{p}}\right) .
$$

(Here $\Omega Y$ denotes the associative model of the loop space on $Y$.) It is immediate from the construction that the $f\left(i_{1}, \cdots, i_{p}\right)$ 's yield a $C_{n}$-form for $f_{1}, \cdots, f_{n}$. This discussion is summarized in the following theorem.

THeOREM 2. If a map $\bar{f}: S Z_{1} \bigvee \cdots \vee S Z_{n} \rightarrow Y$ extends to $F: S Z_{1}$ $\times \cdots \times S Z_{n} \rightarrow Y$, then there exists a $C_{n}-$ form $f\left(i_{1}, \cdots, i_{p}\right)$ for the maps $f_{1}, \cdots, f_{n}$ obtained as above.

To return to the general case, let $X_{1}, \cdots, X_{n}$ be spaces, $g_{i}: X_{i} \rightarrow Y$, $i=1, \cdots, n$, be maps, and $g: X_{1} \vee \cdots \vee X_{n} \rightarrow Y$ be defined by $g \mid X_{i}=g_{i}$. Suppose that $g$ extends to $G: X_{1} \times \cdots \times X_{n} \rightarrow Y$. Then for any suspensions $S Z_{1}, \cdots, S Z_{n}$ and maps $\phi_{i}: S Z_{i} \rightarrow X_{i}$, the composition $\tilde{f}$ given by

$$
S Z_{1} \vee \cdots \vee S Z_{n} \stackrel{\phi_{1} \vee \cdots \vee \phi_{n}}{\longrightarrow} X_{1} \vee \cdots \vee X_{n} \stackrel{g}{\rightarrow} Y
$$

extends to $F: S Z_{1} \times \cdots \times S Z_{n} \rightarrow Y$. Thus the following corollary holds.

Corollary 3. Let $g: X_{1} \bigvee \cdots \vee X_{n} \rightarrow Y$ be a map. If $g$ extends to a map $G: X_{1} \times \cdots \times X_{n} \rightarrow Y$, then for any choice of spaces and maps $\phi_{i}: S Z_{i} \rightarrow X_{i}$, there is a $C_{n}$-form for the maps $f_{i}: Z_{i} \rightarrow \Omega Y$ obtained as above.

REMark A. Although the corollary may seem rather unwieldy, in practice there is always a good candidate for the $\phi_{i}$, namely the evaluation map $e_{i}: S \Omega X_{i} \rightarrow X_{i}$. For this choice, the maps $f_{i}$ are just the looped maps $\Omega g_{i}: \Omega X_{i} \rightarrow \Omega Y$. 
REMARK B. In [5], the higher Whitehead product set $\left[f_{1}, \cdots, \tilde{f}_{n}\right]$ was defined, which measured the obstructions to extending $\tilde{f}$ to $F$ as in Theorem 2. Analogously, a higher Samelson product set $\left\langle f_{1}, \cdots\right.$, $\left.f_{n}\right\rangle$ may be constructed which measures the obstructions to the existence of a $C_{n}$-form for $f_{1}, \cdots, f_{n}$. In a future paper, the author will construct $\left\langle f_{1}, \cdots, f_{n}\right\rangle$ and examine its relationship to $\left[f_{1}, \cdots, f_{n}\right]$ and to the higher homology commutator products discussed below.

2. In order to transfer our situation to homology groups wherein calculations can be carried out, it is necessary to introduce the homology version of higher homotopy commutativity. Let $G$ be an associative $H$-space.

Definition 4. Let $a_{i} \in C_{n_{i}}(G), i=1, \cdots, n$, be cycles. Then a determining system for a higher commutator product of the $a_{i}$ 's is a system of elements $a\left(i_{1}, \cdots, i_{p}\right)$ of $C_{*}(G)$, indexed by proper subsequences of $(1, \cdots, n)$, such that

$$
a(i)=a_{i},
$$

and

(2)

$$
\partial a\left(i_{1}, \cdots, i_{p}\right)
$$

$$
=\sum_{\left(A_{r}, B_{s}\right)}(-1)^{e\left(A_{r}, B_{s}\right)} a\left(i_{A(1)}, \cdots, i_{A(r)}\right) \quad a\left(i_{B(1)}, \cdots, i_{B(s)}\right),
$$

where the summation is taken over all shuffles of $(1, \cdots, p)$ and

$$
\begin{aligned}
\epsilon\left(A_{r}, B_{s}\right)= & \operatorname{sgn}\left(A_{r}, B_{s}\right)+(s-1)\left(1+\operatorname{deg} a\left(i_{A(1)}, \cdots, i_{A(r)}\right)\right) \\
& +\sum_{B(j)<A(k)} \operatorname{deg} a\left(i_{B(j)}\right) \operatorname{deg} a\left(i_{A(k)}\right) .
\end{aligned}
$$

The higher commutator product determined by this system is the homology class of the cycle

$$
\begin{aligned}
& \bar{a}(1, \cdots, n) \\
= & \sum_{\left(A_{r}, B_{s}\right)}(-1)^{\epsilon\left(A_{r}, B_{s}\right)} a(A(1), \cdots, A(r)) \quad a(B(1), \cdots, B(s)) .
\end{aligned}
$$

By checking the various signs involved, it is easy to verify that $\bar{a}(1, \cdots n)$ is, in fact, a cycle. The sign is chosen to make Proposition 5 below hold. Observe that if $a_{1}, \cdots, a_{n}$ are all odd-dimensional, then all the signs involved are +1 .

The set of all elements obtained in this way from $a_{1}, \cdots, a_{n}$ is called the higher commutator product set of $a_{1}, \cdots, a_{n}$ and denoted by 
$\left\langle a_{1}, \cdots, a_{n}\right\rangle$. It is a subset of $H_{N}(G)$, where $N=\sum_{i=1}^{n} \operatorname{deg} a_{i}+n-2$, is natural with respect to homomorphisms of monoids, and depends only upon the homology classes of $a_{1}, \cdots, a_{n}$.

Now let $Z_{1}, \cdots Z_{n}$ be spaces and $f_{i}: Z_{i} \rightarrow G, i=1, \cdots, n$, be maps. For each $i$, let $\alpha_{i}$ be an element of $H_{*}\left(Z_{i}\right)$ and $a_{i}=f_{i_{*}}\left(\alpha_{i}\right)$. The next proposition follows from Definitions 1 and 4.

Proposition 5. In the above situation, if there exists a $C_{n}$-form for the maps $f_{1}, \cdots, f_{n}$, then 0 is an element of $\left\langle a_{1}, \cdots, a_{n}\right\rangle$.

Proof. Define $a\left(i_{1}, \cdots, i_{p}\right)=f\left(i_{1}, \cdots, i_{p}\right) \nRightarrow\left[\kappa_{p} \otimes \alpha_{i_{1}} \otimes \cdots\right.$ $\left.\otimes \alpha_{i p}\right]$. Here $\kappa_{p}$ is the fundamental cell defined in [3]. The signs needed come out of the formula for $\partial\left(\kappa_{p}\right)$ given in [3] and from the usual signs involved in permuting factors in tensor products.

Note. A consequence of Proposition 5 is that if $G$ is a $C_{n}$-space, then all $\left\langle a_{1}, \cdots, a_{k}\right\rangle, k \leqq n$, contain 0 . The commutator product version of Lemma 20 of [2] holds in this case to guarantee that if $a_{1}, \cdots$, $a_{n+1}$ are all odd-dimensional, then $\left\langle a_{1}, \cdots, a_{n+1}\right\rangle$ consists of a single element.

The following criterion now results from combining Corollary 3 with Proposition 5.

THEOREM 6. Suppose the following conditions hold:

(1) $g: X_{1} \vee \cdots \vee X_{n} \rightarrow Y$ is a map and $g_{i}=g \mid X_{i}, i=1, \cdots, n$;

(2) $Z_{1}, \cdots, Z_{n}$ are spaces and $\phi_{i}: S Z_{i} \rightarrow X_{i}, i=1, \cdots, n$, are maps;

(3) for $i=1, \cdots, n, f_{i}: Z_{i} \rightarrow \Omega Y$ is the adjoint to $\tilde{f}_{i}=g_{i} \circ \phi_{i}: S Z_{i}$ $\rightarrow Y$; and

(4) for $i=1, \cdots, n, \alpha_{i} \in H_{*}\left(Z_{i}\right)$ and $a_{i}=f_{i_{*}}\left(\alpha_{i}\right)$.

Then if $g$ extends to $G: X_{1} \times \cdots \times X_{n} \rightarrow Y, 0$ is an element of $\left\langle a_{1}, \cdots, a_{n}\right\rangle$.

REMARK 7. From an examination of the proof of Proposition 5, we see that if $g$ extends to the cartesian product, then 0 is actually an element of the subset $S$ of $\left\langle a_{1}, \cdots, a_{n}\right\rangle$ consisting of those higher product elements whose defining systems are made up of elements which possibly might be obtained as images of maps $f\left(i_{1}, \cdots, i_{p}\right)_{\#}$.

The next section gives an illustration of how this criterion may be applied to a particular extension problem.

3. Denote by $G_{n, k}$ the complex Grassmann manifold of $k$-planes in complex $(k+n)$-space. Given an integer $k$, consider the inclusion $i: G_{k, k} \subset G_{3 k-1},{ }_{3 k-1}$. Let a map $g: G_{k, k} \bigvee G_{k, k} \bigvee G_{k, k} \rightarrow G_{3 k-1,3 k-1}$ be defined to be $i$ on each component. Let $j: G_{3 k-1,3 k-1} \subset G_{3 k, 3 k}$ be the 
inclusion. Then it is well known that $j g$ can be extended to map $G_{k, k} \times G_{k, k} \times G_{k, k}$ into $G_{3 k, 3 k}$ (this is the way the multiplication is defined to make $B U$, the classifying space for the infinite unitary group, into an $H$-space). Here it will be shown that this is the best possible dimension, i.e. that $g$ itself does not extend.

To recreate the situation of Theorem 6 , let $Y=G_{3 k-1}, 3 k-1, X_{i}$ $=G_{k, k}, Z_{i}=S^{2 k-1}$, and $\phi_{i}: S^{2 k} \rightarrow X_{i}$ represent a generator of $\pi_{2 k}\left(G_{k, k}\right)$, for $i=1,2,3$. Thus in this case, each $f_{i}$ represents a generator of $\pi_{2 k-1}(\Omega Y)$. We choose each $\alpha_{i} \in H_{*}\left(S^{2 k-1}\right)$ to be the generator in dimension $2 k-1$, thus $a_{i}=f_{i_{*}}\left(\alpha_{i}\right)$ is known to be $(k-1)$ ! times the generator of $H_{2 k-1}(\Omega Y)$. In order to compute the higher product set $\left\langle a_{1}, a_{2}, a_{3}\right\rangle$, we need a good chain complex at hand to give the homology of $\Omega Y$. The cobar construction fills the bill.

Recall that the ring structure of $H^{*}\left(G_{l, m}\right)$ is obtained by taking the graded ring of formal power series $\Gamma$ on generators $\sigma_{1}, \cdots, \sigma_{m}$, $\operatorname{dim}\left(\sigma_{i}\right)=2 i$, and adding the relations $\tilde{\sigma}_{i}=0, i>l$, defined as follows. Set $\sigma=1+\sigma_{i}+\cdots+\sigma_{m}$, let $\tilde{\sigma}$ be the formal inverse of $\sigma$ in $\Gamma$, and then let $\tilde{\sigma}_{i}$ be the component of $\tilde{\sigma}$ of degree $2 i$.

The cobar construction for $H_{*}(\Omega Y)$ is begun by forming the tensor algebra on generators $\{u\}$ in dimensions one lower than the generators $\{t\}$ of $H_{*}(Y)$. The differential operator, $d$, is defined as follows. Let $\Delta$ be the comultiplication in $H_{*}(Y)$. If $u$ corresponds to $t$, then define $d(u)=\sum u_{i} \otimes u_{i}^{\prime}$, where $\Delta(t)=\sum t_{i} \otimes t_{i}^{\prime}$ and the $t_{i}$ and $t_{i}^{\prime}$ correspond to $u_{i}$ and $u_{i}^{\prime}$ respectively. The complex thus obtained is chain equivalent to the singular chain complex of $\Omega Y$, cf [1].

In $H_{*}(Y)$, let $t\left(i_{1}, \cdots, i_{p}\right)$ denote the element dual to $\sigma_{i_{1}} \sigma_{i_{2}} \cdots \sigma_{i_{p}}, i_{1} \leqq i_{2} \leqq \cdots \leqq i_{p}$. In the cobar construction, let $u\left(i_{1}, \cdots, i_{p}\right)$ correspond to $t\left(i_{1}, \cdots, i_{p}\right)$. A straightforward computation yields that at the chain level, there is a nonzero integer $b$ such that $\left\langle u\left(i_{1}\right), \cdots, u\left(i_{p}\right)\right\rangle=b \cdot d\left(u\left(i_{1}, \cdots, i_{p}\right)\right)$, if $\operatorname{deg}\left(\left\langle u\left(i_{1}\right), \cdots, u\left(i_{p}\right)\right\rangle\right)<6 k-2$. Now each element $a_{i}$ is represented at the chain level by $(k-1)$ ! times $u(k)$. The determining system induced by the multiplication map $G_{k, k} \times G_{k, k} \rightarrow G_{2 k, 2 k}$ yields the element $v=6(k-1) !(u(k, k) u(k)+u(k) u(k, k))$ as an element of $\left\langle a_{1}, a_{2}, a_{3}\right\rangle$. The relation $\tilde{\sigma}_{3 k}=0$ implies that this element is not a boundary. According to Remark 6, we need only consider elements of $\left\langle a_{1}, a_{2}, a_{3}\right\rangle$ which arise from maps $K_{2} \times S^{2 k-1} \times S^{2 k-1} \rightarrow \Omega Y$. But any such element differs from $v$ by a multiple of the element $(k-1)$ ! $(2 k-1) ! \cdot(u(k) u(2 k)+u(2 k) u(k))$, and again, the relation $\tilde{\sigma}_{3 k}=0$ implies that no such element is a boundary. Hence $g$ does not extend to the cartesian product. 


\section{REFERENCES}

1. J. F. Adams, On the cobar construction, Proc. Nat. Acad. Sci. U.S.A. 42 (1956), 409-412. MR 18, 59.

2. D. Kraines, Massey higher products, Trans. Amer. Math. Soc. 124 (1966), 431449. MR 34 \#2010.

3. R. J. Milgram, Iterated loop spaces, Ann. of Math. (2) 84 (1966), 386-403. MR 34 \#6767.

4. G. J. Porter, Higher order Whitehead products and Postnikov systems, Illinois J. Math. 11 (1967), 414-416.

5. - Higher-order Whitehead products, Topology 3 (1965), 123-135. MR 30 \#4261.

6. H. Samelson, $A$ connection between the Whitehead and the Pontryagin product, Amer. J. Math. 75 (1953), 744-752. MR 15, 731.

7. F. D. Williams, Higher homotopy-commutativity, Trans. Amer. Math. Soc. 139 (1969), 190-206. MR 39 \#2163.

New Mexico State University, Las Cruces, New Mexico 88001 